\title{
Design, prototyping, and evaluation of a collapsible device for single-operator sheathing of ultrasound probes
}

\author{
S. A. Lopez $^{1}$, L. R. Hernley ${ }^{1}$, E. N. Bearrick ${ }^{1}$, L. M. Tanenbaum ${ }^{2}$, M. A. C. Thomas ${ }^{1}$, T. A. Toussaint ${ }^{1}$, \\ J. J. Romano ${ }^{3}$, N. C. Hanumara ${ }^{1}$, and A. H. Slocum ${ }^{1}$ \\ ${ }^{1}$ Mechanical Engineering Dept., Massachusetts Institute of Technology, Cambridge, MA, USA \\ ${ }^{2}$ Health Sciences and Technology Dept., Massachusetts Institute of Technology, Cambridge, MA, USA \\ ${ }^{3}$ Hospital Medicine, Massachusetts General Hospital, Boston, MA, USA \\ Correspondence to: S. A. Lopez (saulopez@mit.edu)
}

Received: 18 April 2013 - Revised: 12 August 2013 - Accepted: 5 September 2013 - Published: 15 January 2014

\begin{abstract}
During interventional ultrasound-guided procedures, sterility is maintained by covering the transducer head and cord with a sterile sheath. The current sheathing technique is cumbersome, requires an assistant to complete, and poses a risk of tangling the probe cord and breaching the sterile barrier. This paper presents the design, proof-of-concept prototyping, and evaluation of a probe holder and cartridge-style, single-use applicator that enables faster, more reliable, single-user sheathing of ultrasound probes, with a decreased risk of compromising sterility.
\end{abstract}

\section{Introduction}

\subsection{Opportunity}

The use of ultrasound guidance for interventional procedures has been shown to improve patient outcomes and decrease complications (Mercaldi et al., 2011). Catheter or needle misplacement can cause up to 4-7 excess days of hospital stay, up to USD 45000 in extra costs, and increased morbidity rates of up to $14 \%$ (Zhan et al., 2004). Ultrasound guidance provides real-time visualization of variations in patient anatomy during technically challenging, otherwise blind procedures, including central venous catheter placement, pleural fluid drainage, and various biopsies (Slawsky et al., 2011). In 2011, over 16 million ultrasound-guided procedures were performed in medical settings in the United States, with the estimated savings per 1000 patients totaling over USD 800000 (Slawsky et al., 2011; Sinno and Alam, 2011).

Sterility and image quality are essential during invasive, ultrasound-guided procedures in which needle insertion into the patient occurs. Sterility is achieved through the application of a sheath to the ultrasound transducer before the start of the procedure (Moore, 2011). This technique is cumbersome, requires an assistant, and poses a risk to maintaining the sterile barrier.

The current sheath application process, detailed in Fig. 1, requires that the probe first be held upright while a thin layer of an acoustic gel is applied to the transducer head. This medium aids in transmitting ultrasound signals to tissues to preserve image quality by minimizing the difference in acoustic impedance and sound velocity between the transducer and the human tissue (Marhofer and Frickey, 2006). The sheath is then unwrapped and gloves donned by the clinician. With the assistant grasping first the probe body, inverting it, and then holding it by the cord, the sheath is pulled over the head, secured with a rubber band, and unfurled over approximately $1 \mathrm{~m}$ of cord. If at any point the probe touches the outside of the sheath, the sheath is scrapped and the procedure begins again. While some doctors suggest a different technique when an assistant is not available, no device exists to facilitate the process (Trotter et al., 2011).

An additional challenge comprises maintaining the layer of acoustic gel between the transducer head and sheath. Poor 


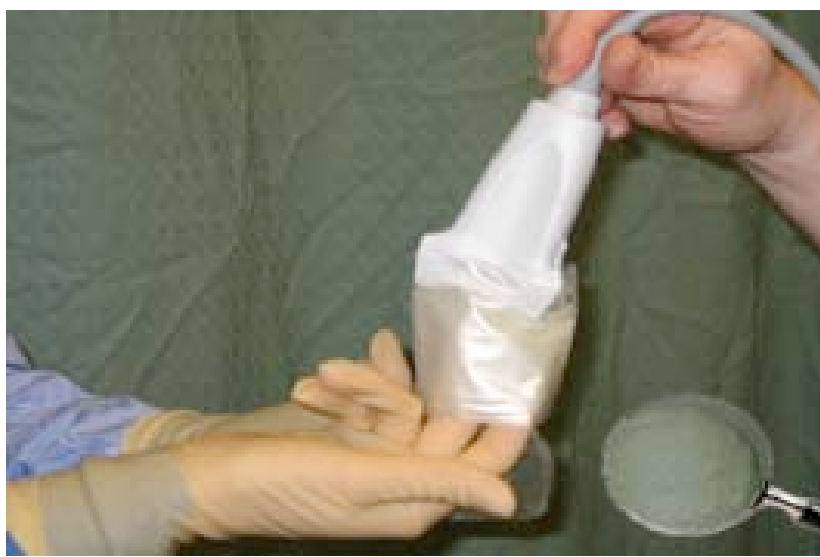

Figure 1. Ultrasound probe shown being sheathed using the current method.

application or its displacement during sheathing can result in air bubbles that reduce image quality.

Given the challenges, the goal of this work was to create a reliable sheathing device for hand-held ultrasound probes that can be operated by a single user.

\subsection{Prior methods}

Several existing devices and patents address sheathing an ultrasound probe; none however, fully address the singleuser scenario. The Pull $\mathrm{Up}^{T M}$ ultrasound cover has a distinct handle-like tab that allows the user to both easily identify the sheath opening and unfurl the sheath over the length of the probe and cord (Witzky, 2012). One patent attempts to increase the ease of unfurling the sheath, often the greatest barrier to maintaining sterility, with a telescoped sheath that is easier to extend along the cord with one hand (US Patent No. 5,910,113, 1999). Additionally, the open end of the sheath is mounted to the outside of a structure that is folded flat; upon use it is opened to ease passage of the probe through the sheath. However, this patent does not adequately eliminate the difficulty of grasping the non-sterile probe while simultaneously applying a sterile sheath. One application broadly addresses covering an ultrasound probe with suggestions for integration of an acoustical couplant (gel replacement) and drawstring sheath-tightening mechanism, without specific solutions (US Patent Application No. 0139944 A1, 2008).

Condom application poses a similar challenge and US patents No. 5,316,019 from 1994 and No. 8,166,975 from 2012 address condom application by a joint packaging of condom and applicator, which extends the condom along the desired length. Sensis ${ }^{\text {TM }}$ tabbed condoms allow the user to unroll the condom with tabs that are freed from the condom when unrolling is complete (DDA Medical, 2009). Both of these products have methods applicable to maintaining sterility because the user holds tabs without having to touch the product surface. The Pronto condom allows direct application of a condom straight from a break-open package (MacDonald, 2011). Stat Strip ${ }^{\circledR}$ adhesive bandages offer similar usability through a bandage that is opened with lateral force and can be applied directly from the packaging (Medline, 2012).

Looking outside the medical field, US patent No. 4,783,950 from 1987 covers protective wrapping of luggage by rotation along its central axis, while enveloping it with a film dispensed from a spool. US patent No. 4,827,695 from 1989 discusses the sealing of a sucker wrapper to a sucker stick through wrapping and heating.

A number of inventions attempt to discourage gel from escaping from the head of the probe. One addresses the issue of fluid frequently escaping by creating a tight fit through an inflatable sheath (US Patent No. 4,815,470, 1989). Another surrounds the ultrasound probe with a homogeneous, solid, elastic, biocompatible sheath that is conformal to each probe and eliminates the need for additional layers of gel (US Patent No. 6,039,878, 2000). Solid couplant hydrogels allow the probe to be placed directly on the patient's skin without the traditional layer of gel on the sheath exterior (US Patent No. $5,522,878,1996)$.

Current devices attempt to improve the handling and application of the sheath and restricting the gel layer. Methods include adding a structure to the sheath and using novel materials for the gel. However, these do not provide a complete solution for a single user to easily grasp the probe while simultaneously applying the sterile sheath.

\section{Design}

A deterministic design process was used that started with determining the functional requirements for the design, progressed from identifying strategies to concepts, and concluded with detailed design and testing (Graham et al., 2007).

\subsection{Functional requirements}

A study of current procedural challenges and device performance constraints resulted in the following functional requirements for a new device:

- Maintain sterility - The device should enable application of a sheath that maintains the sterile barrier between the non-sterile transducer and the patient.

- Single user operation - Eliminate the need for an assistant to manipulate the non-sterile probe and cord. This will reduce procedure costs and, potentially, time.

- Maintain gel layer - Device should facilitate $\sim 3 \mathrm{~mm}$ layer of gel application.

- Cord coverage - Sheath spans $\sim 1 \mathrm{~m}$ of cord. 


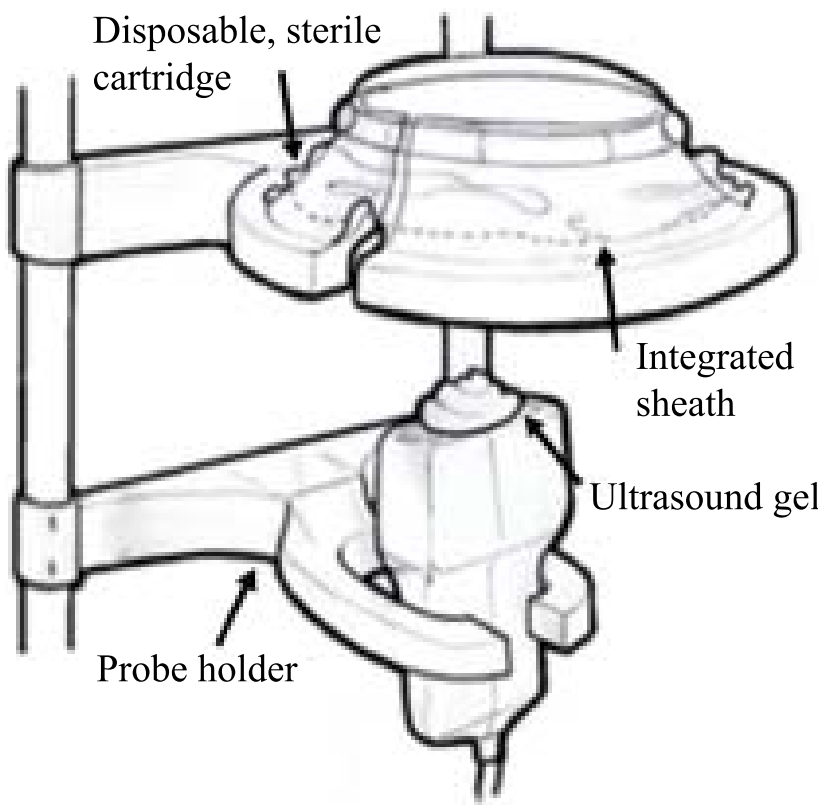

Figure 2. Design concept sketch.

- Easy to use - Reducing the complexity of the transducer sheathing process will ensure repeatable performance and minimize incidents of sterility loss.

- Multiple probe types - Device should accommodate transducers that vary in size, shape, and weight.

- Portable - Device must be portable in order to be transferred across rooms as necessary.

\subsection{System overview}

Shown in Fig. 2 is the two-part concept design consisting of a non-sterile probe holder and a sterile, disposable sheath cartridge that first snaps over the probe, thus protecting the outside of the sheath from contact with the probe. Handles support the rim of the cartridge so it can be manipulated with bare hands. Subsequently, while the clinician wears gloves, the probe is grasped through the sheath and pulled upwards, thus preventing contact between the gloves and the nonsterile probe as the sheath unfurls along the length of the cord. The cartridge then opens to release the now sheathed probe. A flow chart comparing the current method with the proposed method is shown in Fig. 3. The new design reduces complexity by providing a cartridge with a single correct method of deployment, which makes the procedure faster by eliminating steps that pose a risk of breaching the sterile barrier. The process can now be accomplished with a single operator at a significantly lower risk of breaking sterility. Details of the design are provided in the following section.
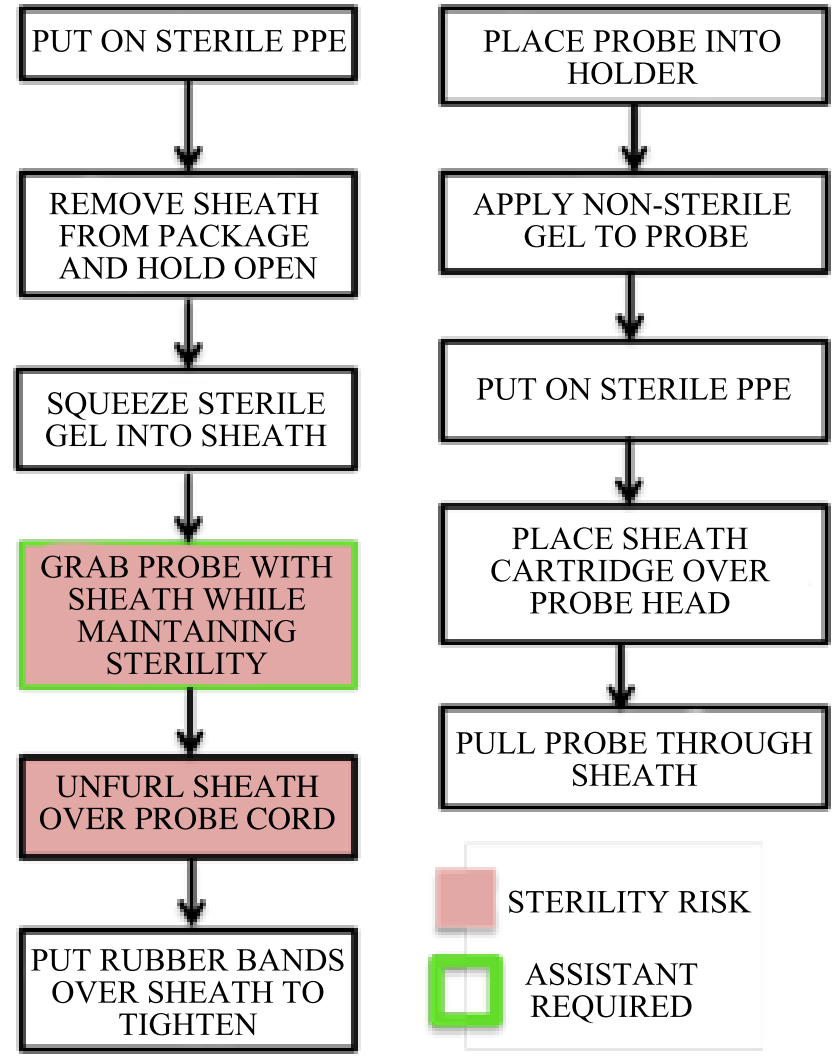

Figure 3. Process flow diagram comparing the current (left) procedure to that using the new device (right).

\section{Module design and analysis}

\subsection{Sheath application \& unfurling}

Applying and unfurling the sheath is the critical part of the procedure that poses the greatest risk to sterility; therefore the most critical module of the device, i.e., sheath application, was designed to eliminate this risk. Bench level experiments and mock-ups demonstrated that fixing the ultrasound probe, rather than the sheath, provided a more ergonomic solution for a single user. Two packaging modalities for the sheath were then considered: traditional telescoping, and rolled, with the latter influencing the first design phase, taking inspiration from Sensis ${ }^{\mathrm{TM}}$ condoms in which tabs are used to directly unroll the condom from the package. Although promising, this technique proved challenging in its implementation and potential manufacturing due to the length of the sheath. Thus, it was decided that incorporating the current telescoping sheath packing would increase the likelihood of the device reaching its market.

The first iteration of this module consisted of the "sheath shell" depicted in Fig. 4. The shell would be packaged and sterilized with the sheath between the inner and outer shell layers, such that the whole assembly could be readily placed over a stationary probe without further sheath preparation. In 


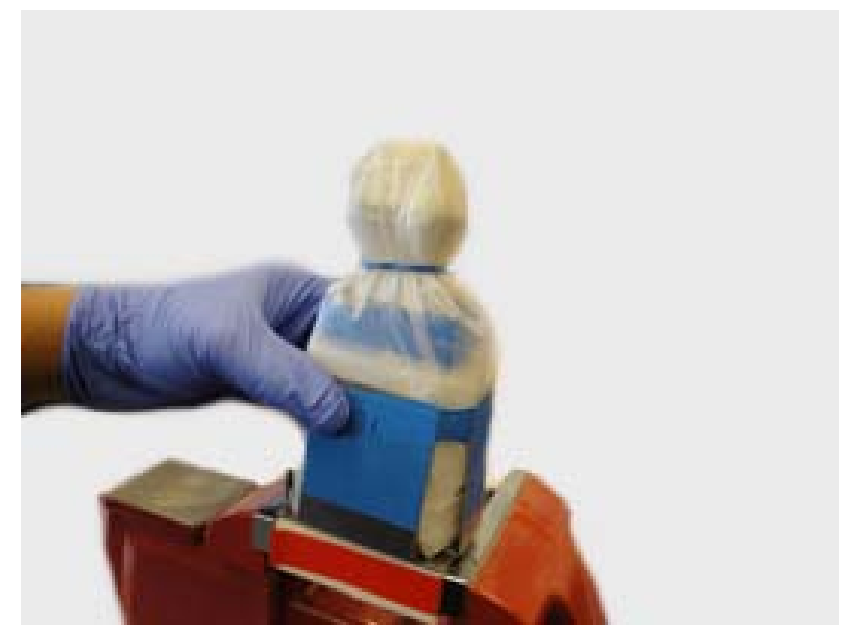

Figure 4. Initial sheath shell prototype showing its placement over a stationary probe.

practice, the gel was applied to the probe head prior to employing the sheath shell, and a rubber band was loaded onto the sheath before the probe was pulled through the shell. The concept is favorable because it creates a clear barrier between non-sterile and sterile procedures. As illustrated in Fig. 3, there is a procedural break where the user can apply sterile personal protective equipment (PPE) before continuing. Additionally, validation testing demonstrated that the device allows the user to easily unfurl the sheath over the length of the cord. The next design iteration addressed cost-effective manufacturing and compact storage of the device.

\subsection{Flat pack design}

The current version of the prototype, illustrated in Fig. 5, addresses the shortcomings of the first sheath shell. For instance, the shell features were optimized to minimize material use, packaging space, and manufacturing complexity. Device compactness was incorporated into the design to minimize the required material and packaged volume. For ease of manufacturing and reducing costs, the sheath shell can be stamped from a sheet of material that can be folded into shape using living hinges (Fig. 6). To keep the design portable, the device folds about the living hinges to a flattened configuration when stored, allowing the use of readily available, sterilizable medical packaging, while keeping the packaged device comparable in size to the current sheathing kits. The implementation of a collapsible sheath shell introduced a number of challenges that were overcome via systematic design and bench-level experimentation. The elements to be addressed included sheath location with respect to the sheath shell, clamping of the bottom of the sheath during the unfurling process, maintenance of sterility, temporary fixation of the sheath shell to the probe holder,

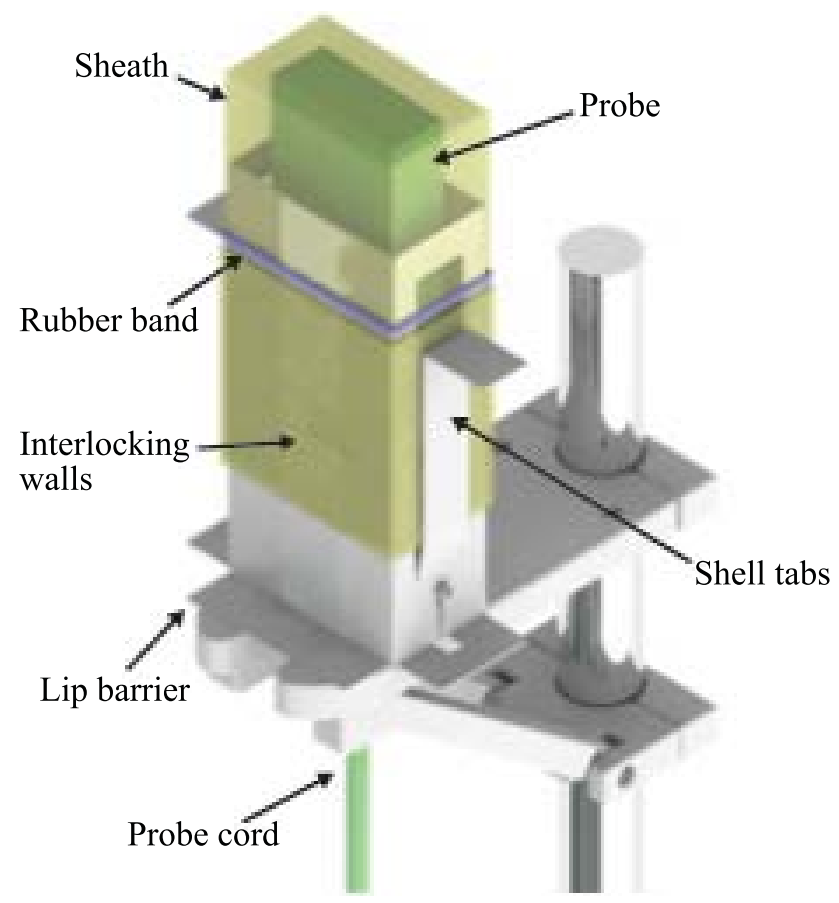

Figure 5. Rendering of the final concept showing sheath shell and integrated probe holder engaged by the sheath shell.

cord removal following sheathing, and rubber band storage and deployment.

In the final design, the sides of the sheath shell contain left and right tabs to allow the user to grasp the shell without compromising sterility or pinching the sheath during the process. A lip was added around the base of the sheath holder to minimize user contact with the non-sterile probe holder. The rubber band is placed on one of the side handles so the user can easily remove it and place it over the probe. The front of the sheath shell has interlocking walls that allow the sheath shell to be rigid while in use yet open following sheathing. The user can pull the probe cord out of the shell while minimizing exposure of the sterile sheath to the nonsterile probe holder. Finally, a snap-fit was included at the rear of the sheath shell to allow the shell to remain fixed to the probe holder while the probe is pulled through the shell for sheathing. Afterwards, the shell is disengaged from the holder and disposed of. The device strongly considers the order of operations of the sheathing procedure and is designed to integrate with existing procedure.

\subsection{Ultrasound probe holder}

The probe holder was designed to mate with the sheath shell as it is placed over the probe. The sheath shell fits over the probe holder to further maintain sterility by enclosing the non-sterile probe within the sterile sheath shell. The holder also contains the complementary parts of the sheath shell snap-fits in order to temporarily fix the sheath shell to the 

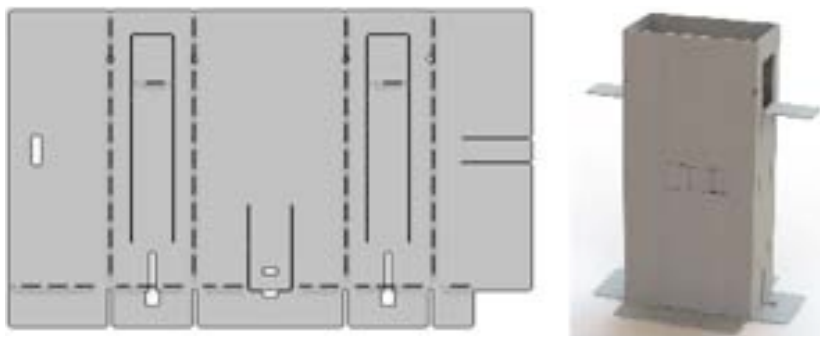

Figure 6. 2-D stamping folded into sheath shell.

probe holder. The probe holder is designed to mount to IV poles, which are readily available in all hospitals and clinics, and in close proximity to portable ultrasound carts. The current prototype was $\mathrm{CNC}$ machined, although it would be injection molded if mass produced.

The collapsible sheath shell design made the incorporation of sterilizable packaging straightforward, as the sheath shell prototype readily fits into the currently used ethylene oxide sterilized easy-open packages, such as the Site Rite* Probe Cover Kit with Gel.

\section{Testing}

\subsection{Repeatability of sheath shell deployment}

The benefit of using a device to constrain the sheath allows the process to be streamlined across users. Thus, repeatability was an important metric of the success of the device. To approximate the reliability of the device, the probe was sheathed 30 times and the process times recorded. A histogram of the results is shown in Fig. 7. In 28 out of the 30 trials, the probe was successfully sheathed as intended. In one trial, the sheath came off the shell without telescoping and in another the snap fit did not engage. Having an adhesive hold the bottom of the sheath could eliminate the risk of the sheath being removed from the shell without telescoping. It should be noted, that the same prototype sheath shell was tested repeatedly, while the design intent is for single use. A more accurate evaluation will be performed once the final sheath shells are manufactured with true living hinges.

\subsection{Sterility}

The ability of the device to maintain sterility is crucial. To ensure that the sheath shell prevented user or sheath contact with the non-sterile probe, cord, or probe holder, the transfer of bacteria from the non-sterile field was simulated through coating the probe with phosphorescent paint and deploying the sheath.

The results shown in Fig. 8 show, as expected, that the transfer of paint was limited to the interior of the sheath shell, which was in direct contact with the probe and holder. The exterior of the sheath shell, the exterior of the sheath, and the

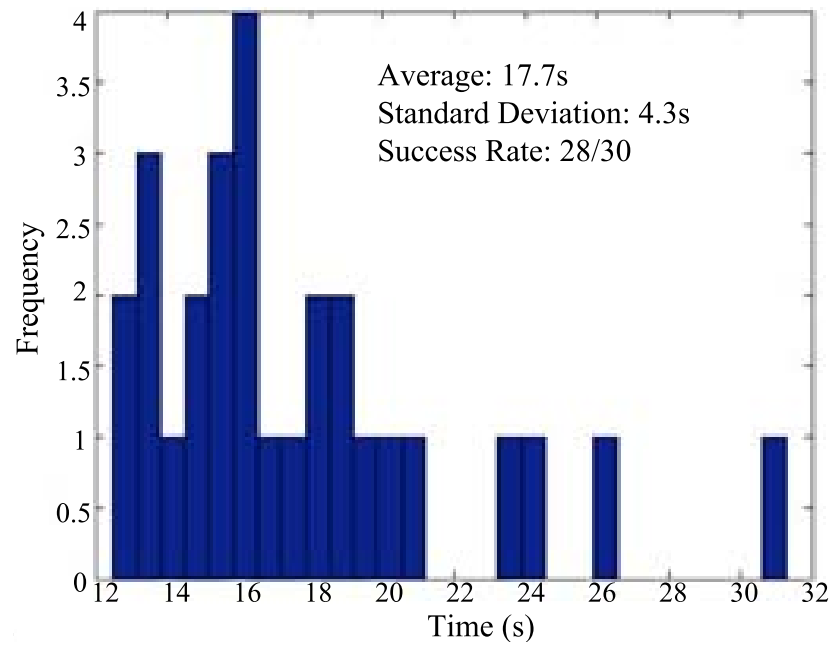

Figure 7. Histogram showing the time distribution of the 30 sheathing trials that were used to approximate the repeatability.

user's gloves were free of the paint. The sterility simulation was performed twice and not tested more extensively for repeatability. As a next step in the development of the device, bacterial culture testing to demonstrate that the sheath and sheath shell are sterile post sterilization will be a crucial step for regulatory approval and product deployment.

\section{Future work}

Although initial testing suggests that the device is reliable, future improvements will be made to ensure that the device is easy to use, maintains good image quality, and is manufactured for clinical use. Two main areas of continued investigation include: a method of automatically deploying the rubber band to secure the sheath to the transducer head, and integrating the gel into the sheath.

\subsection{Rubber band deployment}

Concept designs that will store a rubber band in an unstressed state, then stretch it out and deploy it onto the probe head are shown in Fig. 9.

It was envisioned that storing the rubber band around the indented part of the profile would allow the rubber band to be packaged on the sheath shell and as the probe holder pushes through the sheath shell, the rubber band would be deployed onto the probe. However, the benefits of manufacturing the device out of a single sheet outweighed the possible success of a device with an integrated rubber band.

For future considerations, the incorporation of a similar rubber band mechanism into the single-sheet design would make our device easier to use.

Eliminating the rubber band altogether by including an elastic sheath or an alternate means of tightening the sheath below the head of the probe will also be considered. 

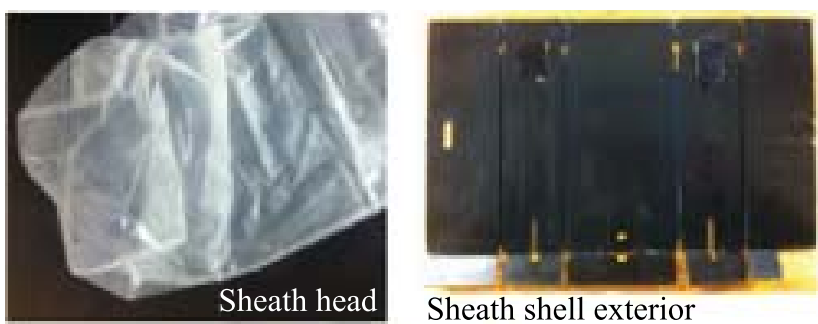

Sheath shell exterior

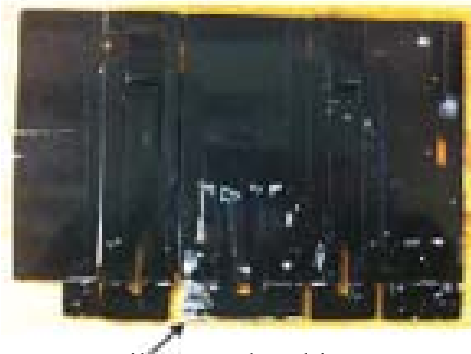

Non-sterile post-sheathing

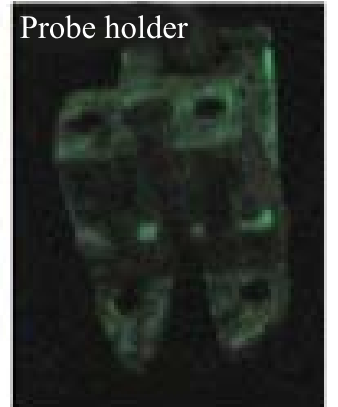

Figure 8. Results of testing with phosphorescent paint coated probe to demonstrate no probe contact outside of the sheath. Top two images show lack of sheath and outer shell contact with probe.

\subsection{Integrated gel}

Air bubbles resulting from the application of gel in the current procedure motivated significant efforts to improve ultrasound image quality through use of a semi-solid gel. Air bubbles are detrimental to image quality because the acoustic impedance and sound velocity of air are very different from that of soft tissue (Marhofer and Frickey, 2006). When considering a medium to better bridge the ultrasound probe and the surface of the body, two requirements must be satisfied: (1) minimize air bubbles within the medium and (2) match impedance and sound velocity to that of human tissues.

A medium that better adheres to the head of the transducer during the sheathing process is desirable in order to maintain image quality throughout the procedure. Ecoflex ${ }^{\circledR}$ Gel silicone was chosen for testing because the properties of silicone closely match those of human tissue. Image analysis using MATLAB ${ }^{\circledR}$ demonstrated that the image depth is comparable, but the resolution of the standard gel is better than that achieved using the silicone. However, future work to minimize bubble formation during the curing of the silicone could offer substantial improvement in resulting image quality. The use of a semi-solid gel would be beneficial to the sheathing process because it eliminates a step in the procedure as well as prevents gel migration from the head of the probe.

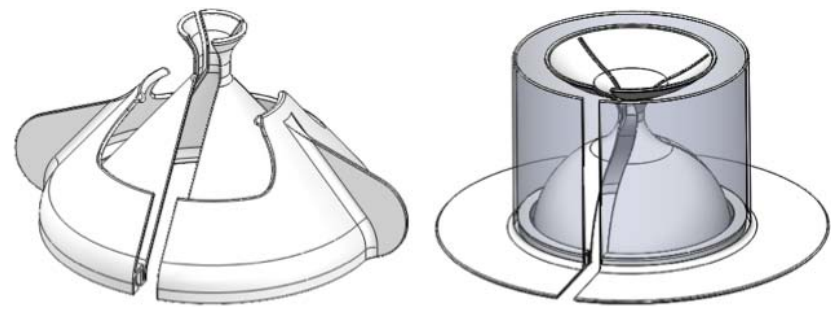

Figure 9. Conceptual designs of a shell with a narrow neck for rubber band storage. As the probe is pushed through, the rubber band is pushed off and snaps onto the probe over the sheath.

\section{Conclusions}

The proposed device satisfies the stated functional requirements. It maintains sterility during the sheathing process, can be operated by a single user, facilitates sheathing of the cord, and is portable. The design of the sheath shell and holder can also be easily modified for fabrication of various ultrasound probe-specific models and the sheath shell can be manufactured from a single sheet, allowing no additional packaging requirements.

Acknowledgements. We would like to thank the Precision Machine Design staff at MIT, as well as those who assisted with the research and testing at the Massachusetts General Hospital.

This work was supported by CIMIT under US Army Medical Research Acquisition Activity Cooperative Agreement W81XWH09-2-0001. The information contained herein does not necessarily reflect the position or policy of the Government and no official endorsement should be inferred.

\section{Edited by: D. Brouwer}

Revied by: two anonymous referees

\section{References}

Curtis, B. B. and Cermak, I. A.: Inflatable Sheath for Ultrasound Probe, USA, 4, 815, 470, 1989.

DDA Medical: Sensis Condoms, available at: http://www. sensiscondoms.com/ (last access: 9 November 2012), 2009.

Graham, M., Slocum, A., and Moreno Sanchez, R.: Teaching high school students and college freshman product development by Deterministic Design with PREP, ASME J. Mechan. Design (Special Issue on Design Engineering Education), 129, 677-681, 2007.

Hanumara, N. C., Begg, N. D., Walsh, C. J., Custer, D., Gupta, R., Osborn, L. R., and Slocum, A. H.: Classroom to Clinic: Merging Education and Research to Efficiently Prototype Medical Devices, IEEE J. Trans. Eng. Health Medic., 1, 2168-2372, 2013.

Jones, K. G.: Condom Applicator, USA, 5, 316, 019, 1994.

Larson, M. J., Rutter, J. W., and Smith, L. L.: Coupling Sheath for Ultrasound Transducers, USA, 6, 039, 694, 2000.

Logan, K. W.: Apparatus for Heat Sealing a Candy Wrapper to a Sucker Stick, USA, 4, 827, 695, 1989. 
MacDonald, J.: Pronto condom aims for global distribution, available at: http://www.changehub.net/2011/10/ pronto-condom-aims-for-global-distribution/ (last access: 7 November 2012), 2011.

Marhofer, P. and Frickey, N.: Ultrasonographic guidance in pediatric regional anesthesia part 1: theoretical background, Pediatric Anesthesia, 16, 1008-1018, 2006.

Medline: Stat Strip Adhesive Bandages, available at: http: //www.medline.com/product/Stat-Strip-Adhesive-Bandages/ Adhesive-Bandages/Z05-PF00037 (last access: 3 October 2012), 2012.

Mercaldi, C. J.: Clinical and Economic Advantage of Ultrasound Guidance Among Patients Undergoing Paracentesis, Technical Report, SonoSite, Bothell, WA, 2011.

Montecalvo, D. A. and Rolf, D.: Solid Multipurpose Ultrasonic Biomedical Couplant Gel in Sheet Form and Method, USA, 5, 522, 878, 1996.

Moore, C.: Ultrasound-guided procedures in emergency medicine, Ultrasound Clinics, 6 277-289, 2011.

Morgan, M.: Combination Prophylactic Package and Dispenser, USA, 8, 166, 975, 2012.

Pruter, R. L.: Sheath for Ultrasound Probe, USA, 5, 910, 113, 1999.

Santagati, U.: Machine for Automatic Protective Wrapping for Use with Different-Sized Baggage, USA, 4, 783, 950, 1987.
Sinno, M. and Alam, M.: Echocardiographically Detected Fibrinous Sheaths Associated with Central Venous Catheters, Echocardiography, 29, E56-E59, 2011.

Slawsky, K., McInnis, M., Goss, T. F., and Lee, D. W.: The clinical economics of ultrasound-guided procedures, Technical Report, General Electric, Wauwatosa, WI, 2011.

Trotter, M., Nomura, J. T., and Sierzenski, P.R.: Single-operator Sterile Sheathing of Ultrasound Probes for Ultrasound-guided Procedures, Academic Emergency Medicine, November 2010, 17, e153 pp., 2011.

Weymer, R. F., Cannon, M. G., Engle, L. A., Klessel, J. S., Lannutti, A. P., Randall, K. S., and Urbano, J. A.: Devices for Covering Ultrasound Probes of Ultrasound Machines, USA, 2008/0139944 A1, 2008.

Witzky, M.: Pull Up Ultrasound Probe Cover Kit, available at: http://www.coneinstruments.com/ pull-up-ultrasound-probe-cover-kit/p/934436/ (last access: 3 November 2012), 2012.

Zhan, C., Smith, M., and Stryer, D.: Incidences, Outcomes and Factors Associated with Iatrogenic Pneumothorax in Hospitalized Patients, [abstract], in: AcademyHealth Annual Research Meeting, 2004, San Diego, Calif. AcademyHealth, 21, abstract no. $1862,2004$. 